\title{
TOMOGRAFIA COMPUTADORIZADA SEM CONTRASTE INTRAVENOSO NO ABDOME AGUDO: QUANDO E POR QUE USAR*
}

\author{
Edison de Oliveira Freire Filho ${ }^{1}$, Paulo Eduardo Marinho de Jesus ${ }^{2}$, Giuseppe D'Ippolito ${ }^{3}$, \\ Jacob Szejnfeld ${ }^{4}$
}

\begin{abstract}
Resumo A tomografia computadorizada sem contraste intravenoso tem sido freqüentemente proposta na avaliação inicial de pacientes com suspeita de abdome agudo, ocupando o espaço de outros métodos diagnósticos. Os autores apresentam uma revisão bibliográfica dos principais aspectos e eficácia da tomografia computadorizada sem contraste intravenoso no diagnóstico de apendicite aguda, cólica nefrética, diverticulite, pancreatite aguda, apendicite epiplóica, pneumoperitônio e obstrução intestinal. Discutem quais as vantagens e limitações desta técnica de exame, bem como seus aspectos práticos.
\end{abstract}

Unitermos: Apendicite; Diverticulite; Abdome agudo; Tomografia computadorizada; Contraste.

Abstract When and why use unenhanced computed tomography in patients with acute abdomen.

The use of unenhanced computed tomography has been frequently recommended for the initial assessment of patients with clinical suspicion of acute abdomen instead of other diagnostic methods. The authors present a review of the literature on the main aspects, advantages, limitations and efficacy of unenhanced computed tomography for the diagnosis of acute appendicitis, renal colic, diverticulitis, acute pancreatitis, primary epiploic appendicitis, pneumoperitoneum and small bowel obstruction. The advantages and limitations of this technique are also discussed.

Keywords: Appendicitis; Diverticulitis; Acute abdomen; Computed tomography; Contrast media.

\section{INTRODUÇÃO}

O uso da tomografia computadorizada (TC) como método de investigação do abdome representa um aprimoramento significativo no diagnóstico de diversas doenças, com conseqüente impacto nas decisões terapêuticas. Isto tem sido particularmente útil na avaliação do abdome agudo, situação na qual, para o tratamento adequado, são fundamentais a precisão e rapidez diagnósticas, fatores estes potencializados pela introdução das técnicas espiral e com multidetectores e pelo conhecimento acumulado na utilização desta ferramenta diagnóstica.

Os bons resultados obtidos com a utilização da TC helicoidal no estudo do pa-

* Trabalho realizado no Departamento de Diagnóstico por Imagem da Universidade Federal de São Paulo/Escola Paulista de Medicina (Unifesp/EPM), São Paulo, SP.

1. Pós-graduando do Departamento de Diagnóstico por Imagem da Unifesp/EPM.

2. Médico Radiologista da Clínica Radiológica de Anápolis e da Clínica da Imagem de Goiânia.

3. Professor Adjunto do Departamento de Diagnóstico por Imagem da Unifesp/EPM.

4. Professor Livre-Docente, Chefe do Departamento de Diagnóstico por Imagem da Unifesp/EPM.

Endereço para correspondência: Dr. Edison de Oliveira Freire Filho. Rua Deputado Zeca Pereira, 170, ap. 202, Bairro Grageru. Aracaju, SE, 49027-040. E-mail: eofilho@ig.com.br

Recebido para publicação em 24/6/2004. Aceito, após revisão, em 13/8/2004. ciente com abdome agudo têm permitido difundir o seu uso, apesar do custo elevado e certa morbidade, quando comparado a outros métodos de imagem como a radiografia simples do abdome e a ultra-sonografia (US). A utilização seletiva de contraste intravenoso (IV) em exames de TC do abdome permite reduzir significativamente estes limites do método e aumentar a sua disseminação e a sua utilidade.

Inúmeros relatos têm demonstrado a grande utilidade da TC abdominal sem injeção intravenosa de meio de contraste no diagnóstico de diferentes condições abdominais agudas, principalmente na identificação de apendicite aguda, diverticulite e de cálculos ureterais ${ }^{(1-31)}$. É importante observar que apesar de certa controvérsia, o uso da TC de abdome sem contraste IV não exclui a possibilidade da implementação posterior da fase contrastada, sempre que considerada pertinente, garantindo-se assim a plena potencialidade do método.

Mais recentemente, o uso da TC sem contraste tem sido proposto como método alternativo à radiografia simples para a avaliação inicial de pacientes com suspeita de abdome agudo, principalmente por sua elevada sensibilidade e especificidade, aliada à sua rapidez e crescente disponibilidade $^{(32)}$.

O objetivo deste trabalho é apresentar uma revisão das aplicações da TC abdominal sem contraste IV, com ênfase na TC helicoidal (ou espiral), suas vantagens e limitações, relacionando as características tomográficas nas principais doenças nas quais essa técnica é utilizada.

\section{TÉCNICA}

Os esforços realizados na tentativa de investigar anormalidades abdominais agudas utilizando-se TC sem a administração intravenosa de meio de contraste iniciaram-se com Malone et al. ${ }^{(5)}$ em 1993, no estudo da apendicite aguda, e Smith et $a l .{ }^{(17)}$ em 1995, na avaliação de pacientes com dor lombar aguda. Posteriormente, esses estudos foram reproduzidos por outros autores, com resultados semelhantes ${ }^{(4,7,9-12,18,19,26,31,33-36)}$.

Nesses estudos subseqüentes, os protocolos aplicados foram de certa forma homogêneos. Nos exames tomográficos com técnica espiral, os seguintes parâmetros foram usados: não utilização do meio de contraste IV ou via oral (VO); colimação de $5 \mathrm{~mm}$ com intervalos de reconstruções 
de $5 \mathrm{~mm}$ e "pitch" variando de 1,0 a $1,6^{(\mathbf{4} \text {, }}$ 7,10-12,18,19,26,31,33,34,36)

Variações nos protocolos foram observadas no estudo de Smith et al. ${ }^{(\mathbf{1 8})}$, que utilizou técnica convencional em parte dos pacientes e realizou reconstruções com 2 $\mathrm{mm}$ de incremento nos exames com técnica espiral; no estudo de D'Ippolito et al. ${ }^{(\mathbf{3})}$, cujos exames foram realizados com técnica convencional com cortes contíguos de 8 a $10 \mathrm{~mm}$ de espessura; no trabalho de Rao et al. ${ }^{(7)}$, que administrou contraste por via retal (VR) para avaliação de pacientes com suspeita de apendicite aguda; e no trabalho de Sommer et al. ${ }^{(19)}$, que avaliou a utilidade de reconstruções coronais oblíquas e de reformatações multiplanares dos ureteres para avaliar a presença de cálculos.

As vantagens da utilização da técnica espiral incluem: curto tempo de exame; a possibilidade da aquisição das imagens em uma única apnéia, eliminando artefatos respiratórios e de movimento, bem como efeitos de volume parcial, o que reduz o risco potencial de não visualização de estruturas como o apêndice cecal ou cálculos ureterais; e a capacidade de gerar imagens reformatadas de alta qualidade ${ }^{(37)}$.

As vantagens potenciais de não utilizar meio de contraste incluem a possibilidade de realizar o exame imediatamente, sem a necessidade de qualquer preparo oral ou jejum, a eliminação dos riscos de reações adversas ao meio de contraste IV e a redução de $\operatorname{custos}^{(4)}$.

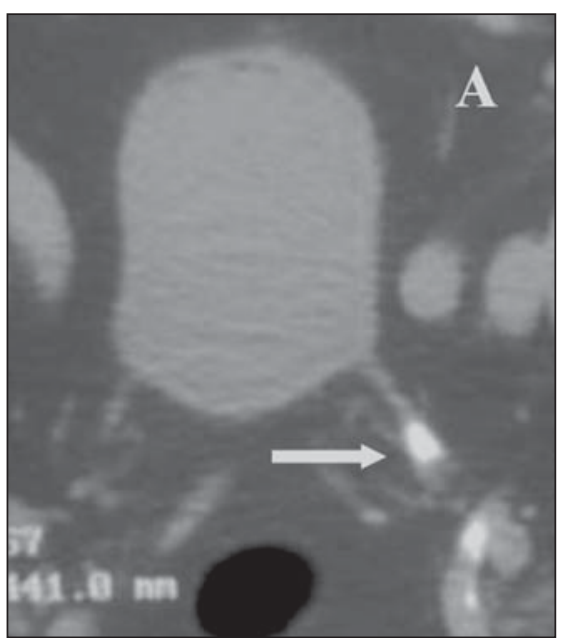

As principais limitações estão relacionadas à incapacidade de se avaliar a função renal, a integridade de órgãos parenquimatosos e estruturas vasculares, e o elevado custo da TC quando comparado com exames alternativos como US e urografia excretora $^{(16)}$. De forma geral, apesar de ainda não comprovado, nota-se a necessidade de uma curva de aprendizado mais longa para a interpretação de exames sem contraste.

Deve-se considerar, entretanto, a capacidade potencial da TC em detectar as diferentes anormalidades que podem resultar em dor abdominal aguda, sendo freqüentemente o único método necessário na maioria desses $\operatorname{casos}^{(\mathbf{1 8 , 3 8})}$. Isto se deve ao elevado poder da TC na descriminação entre tecidos com densidade de partes moles, o que permite excelente avaliação dos órgãos e estruturas intracavitárias, com uma visão panorâmica ${ }^{(3)}$, e fornecendo diagnósticos alternativos (Figuras 1 e 2).

De maneira geral, podemos dizer que os exames tomográficos sem contraste IV ou VO em pacientes com suspeita clínica de abdome agudo dividem-se em duas categorias: 1) com contraste VR, indicado na suspeita de apendicite, diverticulite aguda, apendicite epiplóica e obstrução intestinal baixa; 2) sem contraste VR, indicado na suspeita de urolitíase, abdome agudo perfurativo e obstrução intestinal alta.

Os exames são realizados preferencialmente com técnica espiral (e mais recen-

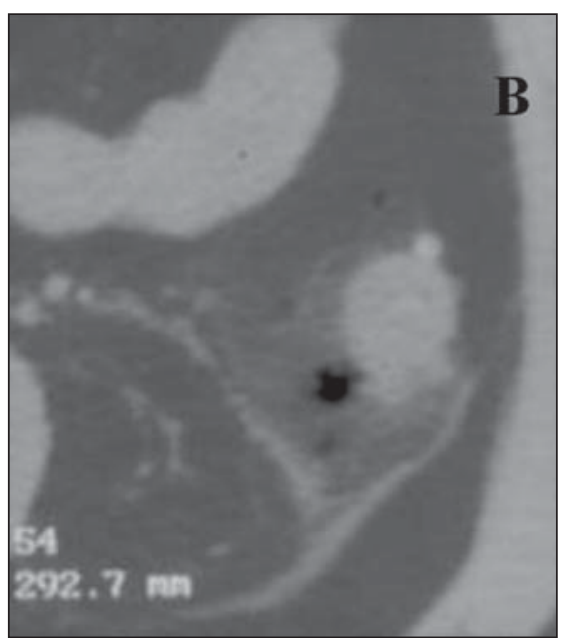

temente com equipamentos "multi-slice"), com cortes contíguos com 5 a $7 \mathrm{~mm}$ de espessura, "pitch" selecionado para obter todos os cortes em uma única apnéia (variando geralmente entre 1,5 e 2), estendendo-se os cortes da cúpula diafragmática até a sínfise púbica. Quando indicado, o contraste VR é introduzido antes do início do exame, com volume que varia entre $500 \mathrm{e}$ $1.000 \mathrm{ml}$ (dependendo da aceitação do paciente) e com diluição de $5 \%$ a $10 \%$. Em casos de dúvida, é recomendável realizar cortes mais finos (2-3 mm de espessura) nas áreas suspeitas. Imagens complementares obtidas em decúbito ventral e lateral podem ser úteis no diagnóstico diferencial de cálculo no ureter distal e vesical, bem como para diagnosticar pneumatose intestinal (Figuras 3 e 4).

\section{APLICAÇÕES}

Até o presente momento, a literatura tem indicado a possibilidade de aplicação da TC sem contraste IV na avaliação da

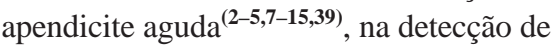
cálculos ureterais e anormalidades não urinárias ocasionando dor lombar agu$\mathrm{da}^{(15-23,25-30,36)}$, na diverticulite aguda ${ }^{(14,15}$ $31,33,40,41)$, no estudo da obstrução intestinal $^{(42-44)}$, da pancreatite aguda ${ }^{(45-49)}$, da apendicite epiplóica $^{(\mathbf{5 0 , 5 1 )}}$ e na detecção de pneumoperitônio ${ }^{(\mathbf{6})}$.

A elevada acurácia diagnóstica da TC sem contraste IV apresentada em alguns es-

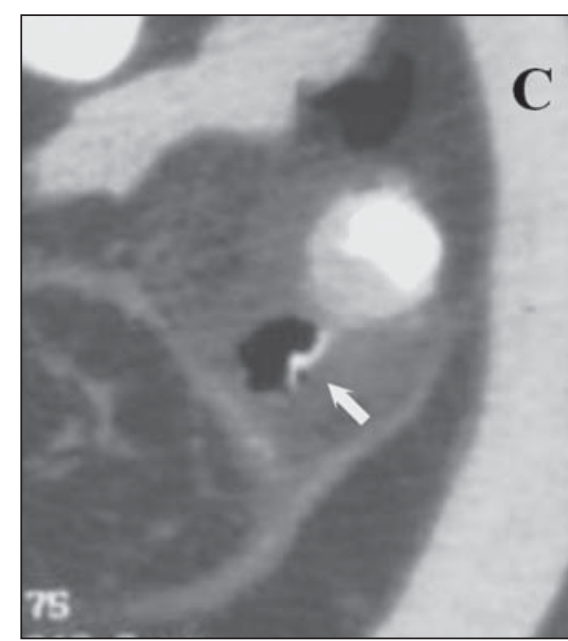

Figura 1. Paciente com cólica nefrética realizou estudo tomográfico sem contraste oral e venoso, em que foi demonstrado, em $\mathbf{A}$, cálculo em ureter distal (seta), e em $\mathbf{B}$, aparente espessamento parietal de cólon descendente, imagem de divertículo, densificação da gordura pericólica, espessamento das fáscias retroperitoneais e ar fora de alça, caracterizando diverticulite aguda, que foi confirmada após uso de contraste retal (C), em que se nota extravasamento do contraste para a coleção gasosa (seta). 
Figura 2. Paciente com suspeita clínica de apendicite aguda realizou tomografia computadorizada sem contraste oral e venoso e com contraste por via retal que demonstrou, em $\mathbf{A}$, apêndice cecal de aspecto normal (seta), e em B, imagem ovalada junto à borda lateral do ceco (seta), com densidade de gordura, porém mais densa que a gordura adjacente, caracterizando apendicite epiplóica cecal.
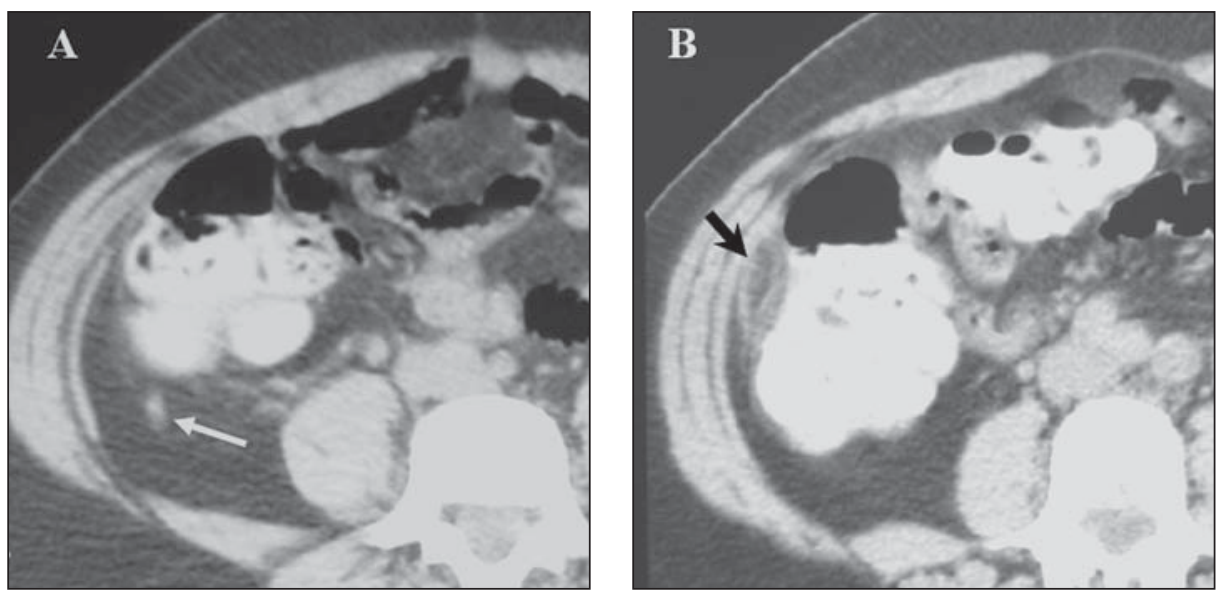

Figura 3. TC de abdome em decúbito dorsal (A) demonstra pequeno cálculo em topografia de meato ureteral direito (seta). Após a mudança de decúbito (B), o cálculo não muda de posição, demonstrando ser realmente um cálculo ureteral e não intravesical.
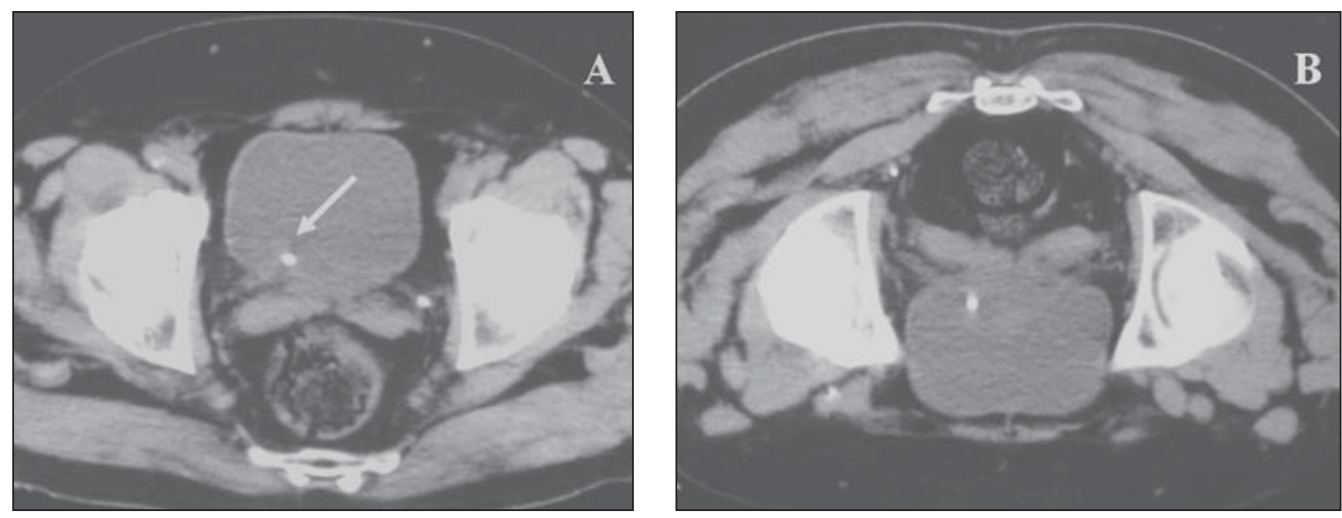
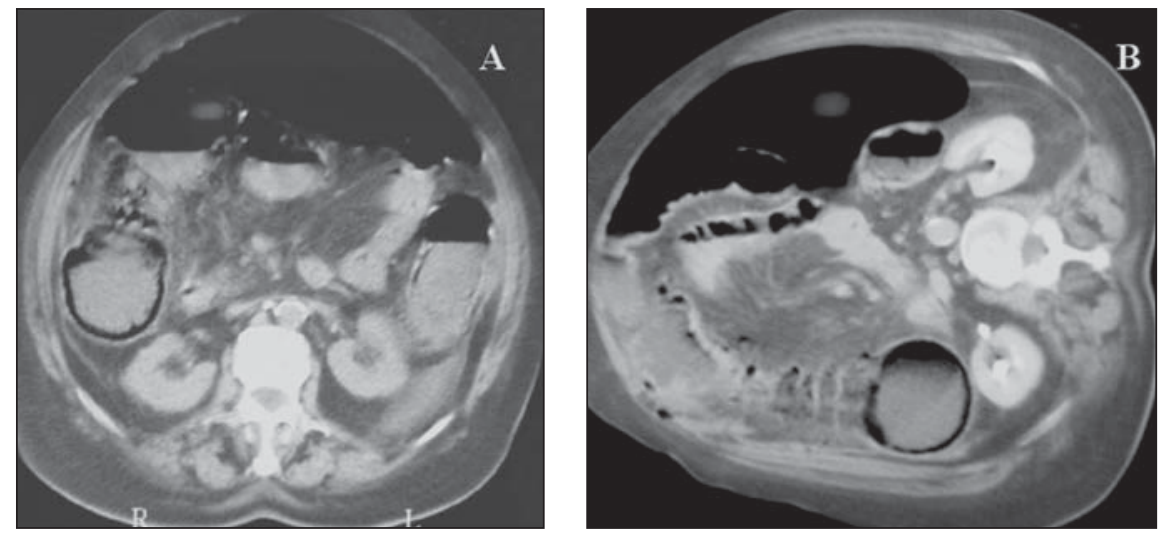

Figura 4. Pneumatose intestinal. Imagens gasosas fixas em parede do cólon ascendente (A), mesmo após mudança para decúbito lateral direito (B).

tudos, associada às vantagens da utilização desta técnica justificam a sua aplicação no abdome agudo, seja como exame inicial, complementar ou mesmo como único exame necessário ${ }^{(\boldsymbol{})}$.

No entanto, as indicações da TC sem contraste IV no abdome agudo têm sido objeto de ampla discussão. Alguns autores advogam o seu uso em detrimento à avaPor outro lado, o elevado custo do exame tomográfico, juntamente com as incertezas em relação aos benefícios de substituir uma técnica tradicional (por exemplo, a radiografia) por esta nova modalidade diagnóstica, têm justificado a cautela na mudança de rotinas e motivado algumas posições divergentes ${ }^{(1,6)}$. liação inicial por radiografia simples ${ }^{(4,18,19)}$.

\section{APENDICITE AGUDA}

Apendicite é um diagnóstico freqüente nos pacientes que procuram os serviços de emergência com dor no quadrante inferior direito do abdome, constituindo a causa mais comum de abdome agudo ${ }^{(52)}$. O diagnóstico geralmente baseia-se nos dados da história, exame físico e testes laboratoriais. Entretanto, até um terço dos pacientes com suspeita de apendicite apresenta quadro clínico e laboratorial atípico $^{(43,53)}$, situação na qual os exames de imagem podem ser imperativos.

O exame ultra-sonográfico tem sido amplamente utilizado no diagnóstico de apendicite aguda, em virtude do seu baixo custo, da sua grande disponibilidade e por ser inócuo, apresentando sensibilidade e especificidade que variam de $75 \%$ a $93 \%$ e de $91 \%$ a $100 \%$, respectivamente ${ }^{(3,54)}$. Todavia, trata-se de um método operadordependente, que requer alta capacitação e experiência do examinador, devendo-se salientar ainda a dificuldade do exame em 
pacientes obesos ou com dor abdominal intensa e naqueles com apêndice retrocecal, quando não é possível implementar a técnica de compressão dosada do apêndice, considerada ideal para otimizar os resultados da US ${ }^{(4,14)}$.

Em 1991, Balthazar et al. ${ }^{(\mathbf{5 5})}$ estudaram, prospectivamente, os exames de TC com contraste IV e VO de 100 pacientes com suspeita de apendicite aguda, encontrando $98 \%$ de sensibilidade, $83 \%$ de especificidade e $93 \%$ de acurácia no diagnóstico de apendicite.

Valores semelhantes foram observados nos relatos de Malone et al. ${ }^{(\mathbf{5})}$ em $1993 \mathrm{e}$ D'Ippolito et al. ${ }^{(3)}$ em 1998, utilizando técnica convencional (não espiral) com cortes contíguos de 8 a $10 \mathrm{~mm}$. Mais recentemente, outros autores, como Lane et al. em $1997^{(\mathbf{4})}$ e em $1999^{(\mathbf{1 0})}$ e Ege et al. ${ }^{(\mathbf{1 2})} \mathrm{em}$ 2002, usando técnica espiral, colimação de 5 mm e "pitch" de 1,6, sem o uso de meio de contraste IV, VO ou VR, demonstraram sensibilidade de $90 \%, 96 \%$ e $96 \%$, especificidade de $97 \%, 99 \%$ e $98 \%$ e acurácia de $94 \%, 97 \%$ e $97 \%$, respectivamente. Segundo Rao et al. ${ }^{(\mathbf{7 , 1 4})}$, a eficácia deste método pode ser otimizada através da utilização de meio de contraste VR, aumentando a sua sensibilidade na detecção de apendicite. Esses autores obtiveram valores de sensibilidade, especificidade e acurácia de $98 \%$. Mullins et al. ${ }^{(11)}$, utilizando esta técnica em crianças, observaram sensibilidade de $97 \%$, especificidade de $99 \%$ e acurácia de $96 \%$.

A TC espiral limitada à transição abdômino-pélvica tem apresentado alta acurácia, reduzindo a apenas um terço a radiação utilizada em exames do abdome completo $^{(7,11,14,56)}$. A contrastação entérica por VO tem sido utilizada em alguns trabalhos ${ }^{(\mathbf{9 , 1 4 , 5 6})}$, porém promove retardo de pelo menos 30 minutos para a realização do exame ${ }^{(\mathbf{8 , 1 4})}$. Também foi demonstrado que o contraste ingerido com uma hora de antecedência alcança o ceco em apenas $50 \%$ dos casos, não sendo útil em parcela considerável de pacientes com suspeita de apendicite aguda $^{(35,57)}$.

O primeiro critério estabelecido por Malone et al. ${ }^{(\mathbf{5})}$ para o diagnóstico de apendicite na TC sem contraste foi o espessamento apendicular superior a $6 \mathrm{~mm}$, com alterações inflamatórias periapendiculares associadas. As presenças de apendicólito e inflamação periapendicular isoladas foram consideradas achados secundários. $\mathrm{O}$ espessamento da fáscia látero-conal foi achado comum e a presença do apendicólito foi considerada muito útil, devendo, contudo, estar associada a alterações inflamatórias do apêndice e planos adiposos pericecoapendiculares $^{(4)}$, para ser valorizada (Figura 5). Outros trabalhos mostraram que o apêndice pode ser considerado normal até $8 \mathrm{~mm}$ de espessura e que mesmo apêndices mais espessos foram encontrados em pacientes normais e assintomáti$\cos ^{(3,35)}$ (Figura 6).

Por outro lado, a ausência de gordura intra-abdominal, bem como a localização pélvica do ceco, demonstraram ser fatores limitantes na interpretação de alguns exames de TC sem contraste ci,5,11) $^{(4)}$

Os diversos estudos que avaliaram o papel da TC sem contraste IV na apendi-
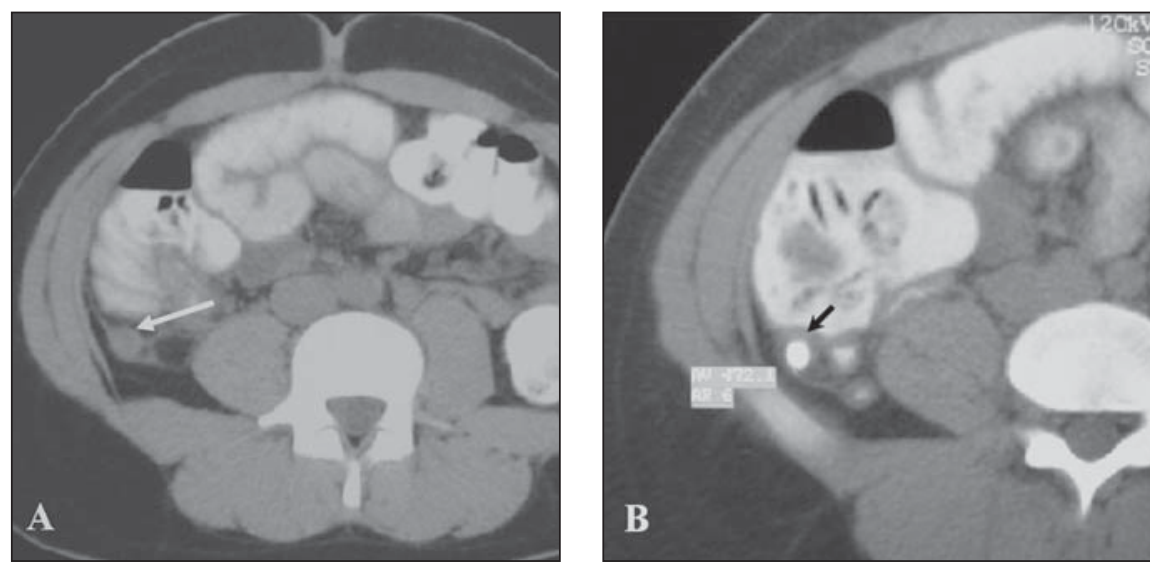

Figura 5. Apendicite aguda. TC de abdome com contraste por via retal e sem contraste venoso demonstra, em A, apêndice cecal espessado com calibre $>6 \mathrm{~mm}$ (seta), associado a densificação da gordura periapendicular e espessamento da fáscia látero-conal direita. Em $\mathbf{B}$ observa-se imagem radiodensa em base do apêndice (seta), representando apendicólito.

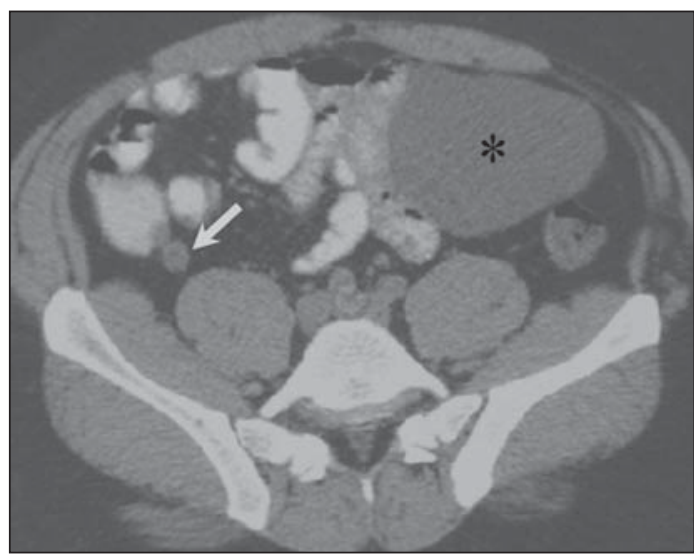

Figura 6. Apêndice cecal com $13 \mathrm{~mm}$ de calibre (seta), sem sinais inflamatórios adjacentes, observado em paciente assintomático em exame de controle de cisto mesentérico (asterisco). 0 aspecto do apêndice era semelhante um ano antes. 
Por aproximadamente 70 anos a urografia excretora foi o método de imagem de escolha para o diagnóstico de ureterolitíase, porém, nos últimos anos, a TC sem contraste com cortes finos e técnica espiral tem ocupado o seu lugar ${ }^{(\mathbf{2 1 - 2 4 , 3 4 )}}$. Os estudos com a utilização desta técnica iniciaram-se em 1995 com Smith et al. ${ }^{(17)} \mathrm{e}$ motivaram trabalhos posteriores, que obtiveram resultados semelhantes e animadores $^{(18,19,22,23,25,26,34)}$.

O diagnóstico tomográfico de litíase ureteral é feito através de sinais diretos, quando se identifica densidade cálcica no interior da luz ureteral ou dentro da bexiga, ou por sinais secundários e indiretos. Os sinais indiretos, em ordem de frequiência, incluem hidroureter, hidronefrose, estriações na gordura perirrenal, aumento do tamanho renal homolateral, parede ureteral visível ao redor do cálculo (sinal do halo) e heterogeneidade periureteral adjacente ao cálculo $^{(\mathbf{2 7 , 3 4 , 3 6 )}}$ (Figura 7). Alguns desses sinais secundários podem estar presentes em alguns pacientes sem doença por cálculo ureteral $^{(\mathbf{3 6})}$, estar ausentes precocemente e tornar-se mais acentuados com o passar do tempo ${ }^{(\mathbf{2 0})}$. Outros achados secundários mais recentemente descritos são a ausência unilateral da pirâmide medular hiperdensa ${ }^{(27)}$ e a redução da atenuação do parênquima renal agudamente obstruído, em comparação com o rim contralateral ${ }^{(\mathbf{2 8})}$ (Figura 7B).

A identificação do cálculo com base na densidade não representa dificuldade. Virtualmente, todos os cálculos apresentamse radiopacos na $\mathrm{TC}$, mesmo os de ácido úrico, que têm densidade variando entre 300 e $400 \mathrm{UH}^{(\mathbf{1 9})}$. As únicas exceções são os cálculos gerados pela deposição de inibidores de protease (Indinavir), que devem ser considerados em pacientes em tratamento para HIV e com sinais obstrutivos indiretos do sistema coletor renal ${ }^{(20,27)}$.

Quando a ureterolitíase está presente, a TC pode determinar precisamente o tamanho e a localização do cálculo. Estas características são utilizadas para avaliar a chance de passagem espontânea e permitem orientar a terapêutica intervencionis$\mathrm{ta}^{(\mathbf{1 8})}$. Hubner et al. ${ }^{\mathbf{( 5 8 )}}$ demonstraram eliminação espontânea de $38 \%$ dos cálculos menores que $4 \mathrm{~mm}$, comparado com apenas $1,2 \%$ dos maiores que $6 \mathrm{~mm}$. Quanto à sua localização nos terços distal, médio e proximal do ureter, esses autores observaram freqüência de eliminação espontânea de $45 \%, 22 \%$ e $12 \%$, respectivamente.

Estudos realizados desde Smith et al. ${ }^{(17)}$ em 1995 obtiveram valores preditivos negativos (VPN) de $91 \%$ a $100 \%$, valores preditivos positivos (VPP) de $96 \%$ a $100 \%$, sensibilidades de $95 \%$ a $100 \%$ e especificidades de $92 \%$ a $100 \%$ para a detecção de cálculos ureterais pela TC espiral sem contraste ${ }^{(23)}$, com excelente reprodutibilidade, independentemente do grau de experiência do radiologista ${ }^{(25,34)}$.

Em virtude do seu elevado VPN, a TC sem contraste pode excluir com segurança a possibilidade de cálculo ureteral e determinar a existência de causas extra-urinárias não suspeitadas de dor abdominal aguda, que incluem apendicite, diverticulite (Figura 1), torção de massa pélvica, aneurisma roto de aorta abdominal, pancreatite, apendicite epiplóica, entre outras
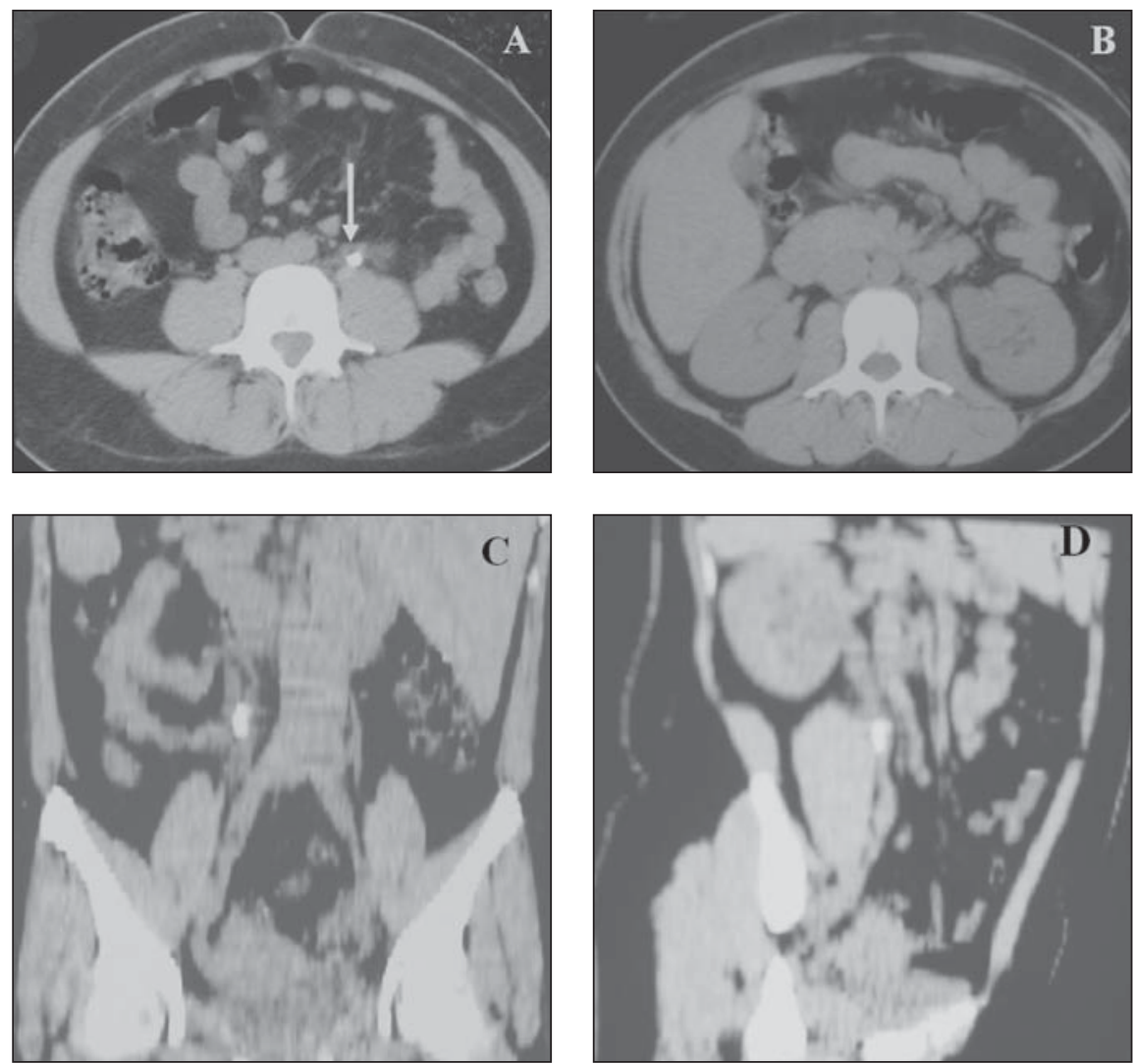

Figura 7. Tomografia computadorizada de abdome sem contraste oral e venoso em paciente com quadro de dor lombar esquerda aguda demonstra, em A, cálculo em terço médio do ureter esquerdo (seta) com espessamento da parede ureteral em volta do cálculo (sinal do halo) e heterogeneidade da gordura periureteral adjacente. Como sinais secundários observam-se, em $\mathbf{B}$, moderada hidronefrose à esquerda $\mathrm{e}$ menor densidade do parênquima renal esquerdo em relação ao contralateral. Reconstruções coronal (C) e sagital oblíqua (D) demonstram bem o trajeto ureteral e o cálculo no seu interior. 
pacientes com pouca gordura retroperitoneal e naqueles sem hidronefrose ou hidroureter. No sentindo de evitar falso-positivos nesses pacientes, todas as calcificações na região do ureter distal devem ser consideradas passíveis de serem cálculos ureterais, e a urografia excretora ou a TC com contraste IV podem ser necessárias em casos selecionados ${ }^{(\mathbf{3 6})}$.

Uma vez que já está estabelecido que a TC sem contraste tem alta eficácia e reprodutibilidade na avaliação de cálculos ureterais, apresenta baixo risco pela não utilização do contraste IV e é de rápida execução, a literatura vem discutindo a sua posição ideal no algoritmo da investigação desta entidade. As principais discussões são em relação à dose de radiação utiliza-

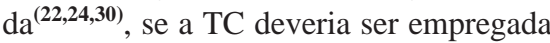
como exame primário, antes mesmo da US e da radiografia simples do abdome ${ }^{(22)}$, e o seu verdadeiro impacto em relação aos custos e ao manejo dos pacientes ${ }^{(\mathbf{2 4})}$

A dose de radiação do estudo tomográfico do abdome para investigação de ureterolitíase é cerca de duas a três vezes maior que a utilizada em uma urografia excretora $^{(23,24)}$. Hamm et al. ${ }^{(23)}$ avaliaram a TC espiral sem contraste com baixa dose de radiação (redução de $50 \%$ a $65 \%$ da dose de exposição), observando sensibilidade de $96 \%$, especificidade de $97 \%$, valores preditivos negativo e positivo de $90 \%$ e $99 \%$, respectivamente, valores semelhantes aos descritos na literatura com a TC espiral com dose de radiação convencional. Sugerem que esta seria a técnica de escolha, com exceção para os pacientes obesos.

Gottlieb et al. ${ }^{\text {(24) }}$ concluem, em seu trabalho publicado em 2002, que o uso dos métodos de imagem em pacientes com suspeita de ureterolitíase vem aumentando significativamente desde a introdução da TC sem contraste, com poucos efeitos sobre os cuidados com os pacientes nos departamentos de emergência. Comparando os períodos antes e após a introdução deste método, observaram taxas semelhantes no percentual de admissões após a primeira consulta, no percentual de retorno ao serviço de emergência e de admissões hospitalares dentro do primeiro mês após a primeira visita, e no tempo de permanência no departamento de serviço de emergência.

\section{DIVERTICULITE AGUDA}

A diverticulite aguda é a complicação mais comum da doença diverticular dos cólons, ocorrendo em $10 \%$ a $20 \%$ dos pacientes com moléstia diverticular conhecida $^{(\mathbf{5 9})}$. O diagnóstico clínico da diverticulite é comumente duvidoso, com relatos de $34 \%$ a $67 \%$ de erros no seu diagnóstico em pacientes de todas as faixas etárias ${ }^{(\mathbf{1 4}, 31)}$. Muitos autores recomendam exames radiológicos precoces de rotina em todos os pacientes com suspeita clínica de diverticulite, devido a essas altas taxas de erro diagnóstico e às suas importantes complicações pré e pós-operatórias ${ }^{(31,33)}$

As radiografias simples só são diagnósticas nos casos muito graves com perfuração intestinal, quando podem ser observa- dos pneumoperitônio ou coleção gasosa pélvica extraluminal ${ }^{(\mathbf{1 , 6 0})}$

Historicamente, pacientes com suspeita clínica de diverticulite têm sido avaliados através de enema opaco. Os achados deste exame incluem identificação de divertículos, hipertrofia da parede muscular, efeito de massa intramural ou extramural sobre a coluna de contraste, obstrução cólica, espasmo cólico e extravasamento intramural ou peritoneal do meio de contraste ${ }^{(\mathbf{1 4 , 1 5})}$. Apesar de ser método bastante sensível e de baixa morbidade quando se utiliza contraste iodado hidrossolúvel, o enema opaco apresenta como principal limitação uma baixa especificidade, não permite avaliar a extensão extracólica da doença e não fornece diagnósticos alterna$\operatorname{tivos}^{(\mathbf{1}, \mathbf{1 4}, \mathbf{1 5}, \mathbf{3 1}, \mathbf{3 3})}$ (Figuras 8, 9, 10 e 11).
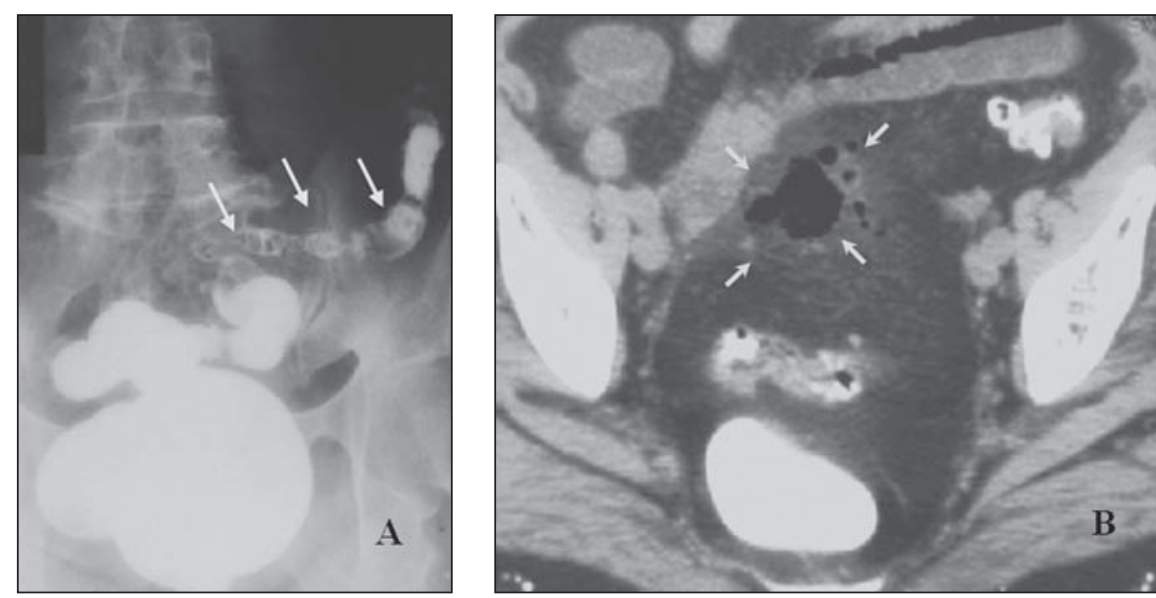

Figura 8. Enema opaco (A) de paciente com dor em fossa ilíaca esquerda demonstra extenso segmento de afilamento irregular do cólon (setas). Estudo tomográfico deste mesmo paciente (B) demonstra coleção gasosa fora de alça (setas), que não foi caracterizada através do enema opaco.
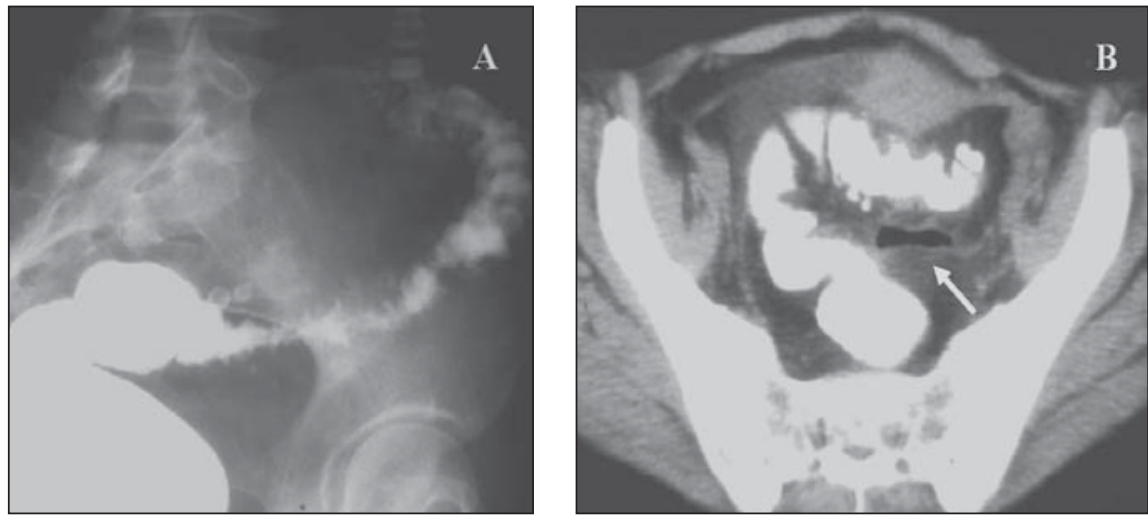

Figura 9. Em A, enema opaco confirmando suspeita clínica de diverticulite aguda caracterizada por extenso segmento de sigmóide afilado e espessamento do relevo mucoso, notando-se algumas imagens de divertículos. Em B, TC sem contraste intravenoso e com contraste por via retal confirma o diagnóstico de diverticulite aguda, mas mostra imagem gasosa fora das alças intestinais (seta), caracterizando perfuração. 

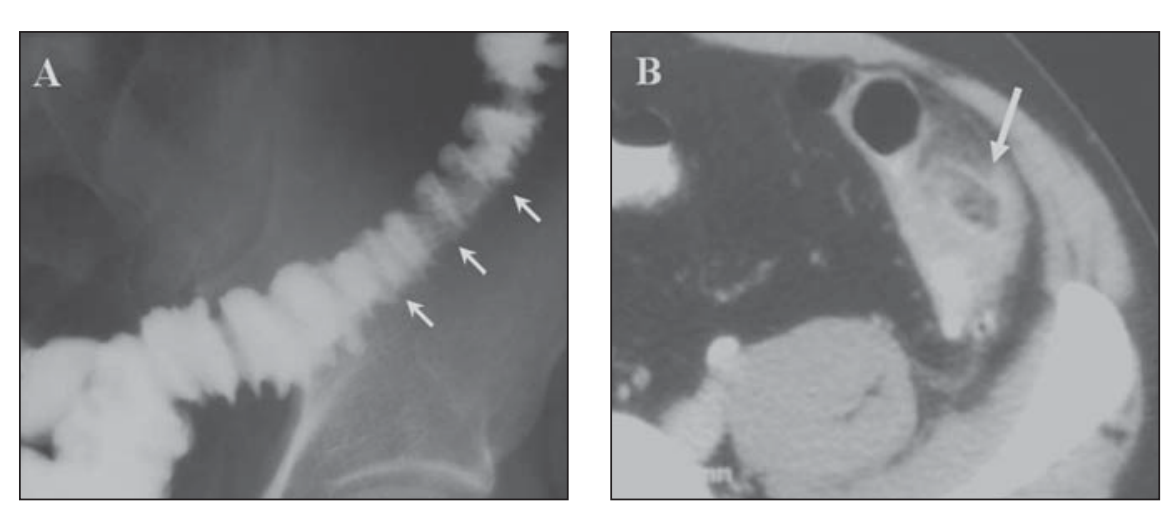

Figura 10. Em A, enema opaco de paciente com dor em fossa ilíaca esquerda e suspeita clínica de diverticulite aguda demonstra retificação da face externa do cólon descendente/sigmóide (setas), sem imagens de divertículo. Em B, estudo tomográfico demonstra imagem ovalada (seta) de densificação da gordura, caracterizando apendicite epiplóica.
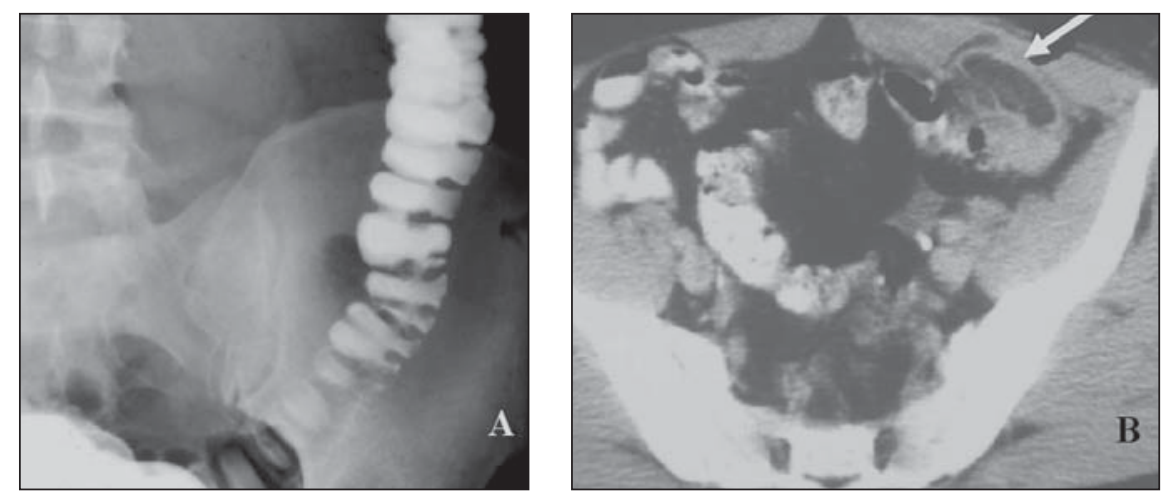

Figura 11. Em A, enema opaco normal de paciente com suspeita clínica de diverticulite aguda. Em $\mathbf{B}$, estudo tomográfico demonstra apêndice epiplóico inflamado (seta) junto à face anterior do sigmóide.

A TC espiral aprimorou dramaticamente o diagnóstico e o manuseio do paciente com diverticulite aguda, substituindo o enema opaco na maioria dos serviços. Este método avalia tanto o componente intramural do processo inflamatório quanto a sua extensão intra e retroperitoneal, permitindo estadiar a doença e assim planejar o tratamento ${ }^{(14,15,31,33)}$.

Achados tomográficos da TC na diverticulite incluem espessamento inflamatório da parede intestinal (maior que $5 \mathrm{~mm}$, com a distensão luminal adequada), densificação da gordura pericólica, bolhas de ar extraluminais, presença de abscessos e líquido livre (Figuras 1, 8, 9 e 12). O extravasamento de contraste também pode ser ob-

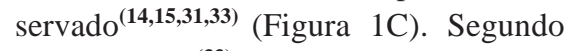
Kircher et $a l .{ }^{(33)}$, os dois sinais mais freqüentes na diverticulite são espessamento parietal $(96 \%)$ e densificação da gordura pericólica (95\%). Sinais menos freqüentes, porém altamente específicos, são espessa-

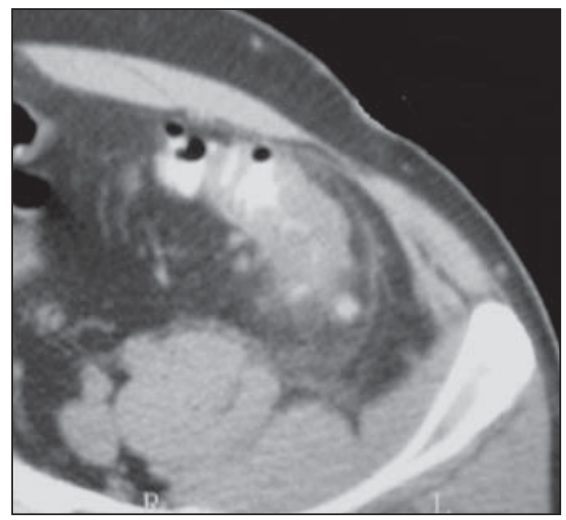

Figura 12. Diverticulite aguda. TC sem contraste intravenoso e com contraste por via retal demonstra espessamento parietal do sigmóide com densificação da gordura adjacente, espessamento da fáscia látero-conal e imagens de divertículos.

mentos das fáscias retroperitoneais (50\%), líquido livre (45\%), divertículo inflamado (43\%) e líquido na raiz do mesocólon.

A TC sem contraste IV e com contraste VR tem-se tornado o método de escolha

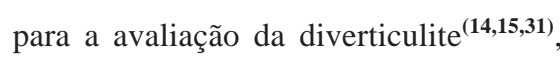
com relatos de até $97 \%$ de sensibilidade, $100 \%$ de especificidade e $99 \%$ de acurácia, observados por Rao et al. ${ }^{(\mathbf{3 1})}$ em 1998.

A distensão cólica pelo contraste VR permite a distinção entre espessamento parietal verdadeiro e espessamento parietal aparente causado pela alça não distendida, e identificação e distinção de ar e coleções líquidas (abscessos) entre intraluminal e extraluminal. O contraste administrado VO não permite avaliação do espessamento parietal cólico, pois este fica parcialmente distendido, além de retardar o exame em pelo menos duas horas. Mesmo após duas horas de preparo, em até $50 \%$ dos casos o contraste não alcança o cólon ${ }^{(35)}$.

O contraste IV aumenta os custos e os riscos, sem adicionar significativamente novas informações ${ }^{(\mathbf{1 4 , 3 3 )}}$. É importante observar, no entanto, que o diagnóstico diferencial com câncer de sigmóide pode ser bastante difícil, sendo necessário, em casos de dúvida, realizar a fase contrastada ou enema opaco e colonoscopia, para diferenciar essas duas doenças ${ }^{(\mathbf{6 1})}$.

\section{PANCREATITE AGUDA}

O diagnóstico da pancreatite aguda é geralmente baseado nos sintomas clínicos e na elevação acima de três vezes do valor normal dos níveis séricos de amilase e li-

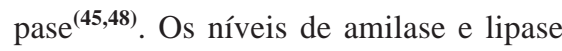
podem ser normais em cerca de $10 \%$ dos pacientes com pancreatite aguda ${ }^{(57)}$. No diagnóstico diferencial encontramos isquemia ou infarto mesentérico, úlcera gástrica ou duodenal perfurada, cólica biliar, entre outros ${ }^{(\mathbf{4 8})}$. Exames de imagem são realizados por várias razões, incluindo a detecção de cálculos biliares, detecção de obstrução biliar, diagnóstico de pancreatite quando a situação clínica não está clara, identificação de pacientes de alto risco e detecção de suas complicações ${ }^{(62)}$.

US deve ser realizada em todo paciente com pancreatite aguda para detectar cálculos na vesícula biliar ou no colédoco, mesmo nos consumidores de álcool. Este método é mais limitado no diagnóstico da coledocolitíase distal e para visualizar o pâncreas e a região peripancreática, devido à ausência de janela acústica pela interposição gasosa ${ }^{(\mathbf{6 2})}$. 
A TC é método pouco sensível para detectar cálculos biliares, mas é excelente para delinear o pâncreas e as anormalidades e complicações associadas à pancreatite $\operatorname{aguda}^{(62)}$.

Uma vez estabelecido o diagnóstico da pancreatite aguda, a intensidade, duração e o tipo de tratamento dependem da definição precoce da gravidade da doença. Essa definição, baseada em parâmetros objetivos, é crucial para predizer complicações clínicas e identificar ataques potencialmente fatais ${ }^{(47,63)}$. Em 1985, Balthazar et al. ${ }^{(\mathbf{6 3})}$ criaram um sistema de graduação de gravidade de pancreatite aguda, com base em alterações inflamatórias peripancreáticas e da morfologia do pâncreas, observadas à TC (índice morfológico). Em 1990 ${ }^{(64)}$, esse mesmo grupo demonstrou, através da TC com injeção dinâmica de contraste IV, haver correlação entre a presença e a extensão de necrose pancreática com o prognóstico da pancreatite aguda. Com a associação dos critérios descritos em 1985 e a avaliação da necrose pancreática, foi criado o índice de gravidade da TC para pancreatite aguda (ISTC) (Figura 13).

Vários autores e consensos internacionais concordam que a presença e a extensão da necrose pancreática são os indicadores mais importantes da gravidade da

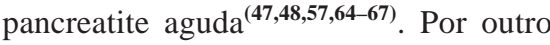
lado, seu papel tem sido questionado por

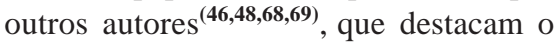
valor das alterações inflamatórias pancreáticas e peripancreáticas no estadiamento da pancreatite aguda ${ }^{(46,48,68)}$.

Em artigo de revisão recente, Baltha$\operatorname{zar}^{(47)}$ afirmou que a necrose pancreática desenvolve-se dentro das primeiras 24-48 horas do início dos sintomas clínicos, que estudos tomográficos realizados precocemente mostram achados equívocos relacionados à isquemia da glândula, que exames realizados após três dias têm maior acurácia diagnóstica, e conclui que, para o estadiamento da pancreatite aguda, a TC somente deveria ser realizada após 48-72 horas do início do quadro clínico. Esta conduta levaria a um retardo no estadiamento da pancreatite aguda.

A TC sem contraste IV tem sido citada na avaliação de alterações peripancreáticas e da morfologia pancreática como alternativa para estratificação da gravidade da
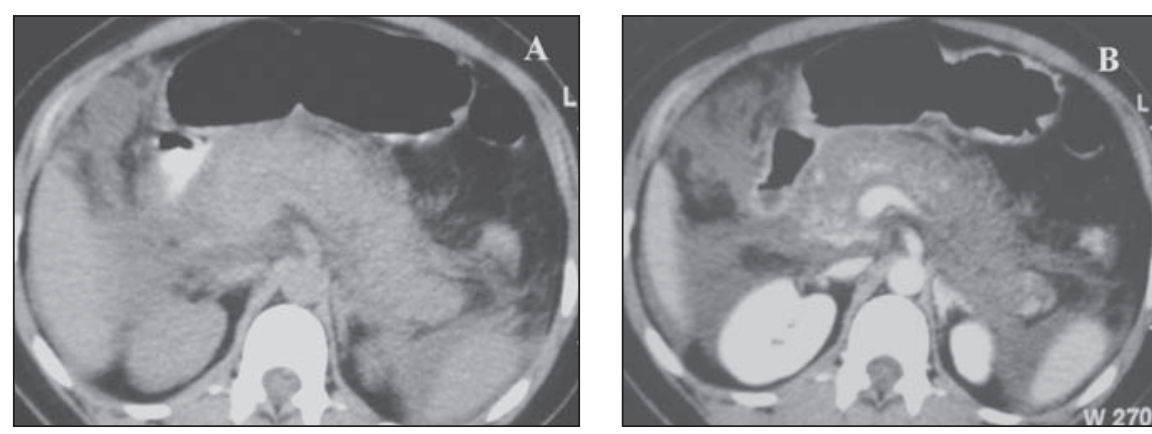

Figura 13. Em A, TC sem contraste de paciente com diagnóstico de pancreatite aguda demonstra pâncreas difusamente aumentado, com alterações inflamatórias peripancreáticas e líquido coletado em espaço pararrenal anterior bilateralmente, periesplênico e intraperitoneal (grau E do índice morfológico de Balthazar). Em B, na fase após a injeção intravenosa do meio de contraste, nota-se necrose acometendo mais de $50 \%$ do parênquima pancreático (ISTC $=10)$.

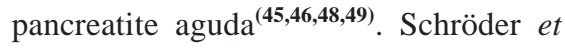
$a l .{ }^{(45)}$ propõem que a TC sem contraste seria suficiente para avaliação de alterações extrapancreáticas na definição de seu escore. De Sanctis et al. ${ }^{(\mathbf{4 6})}$, após definirem no seu estudo que as coleções inflamatórias peripancreáticas aparentam ter maior valor prognóstico que a necrose pancreática, concluem que a TC sem contraste seria adequada para definir complicações locais e guiar intervenções na maioria dos casos. Mais recentemente, Balthazar ${ }^{(47)}$, ao dissertar sobre o índice morfológico criado por seu grupo em $1985^{(63)}$, afirma que para sua obtenção não é necessária a utilização do meio de contraste IV (Figuras 13 e 14).

Dois estudos avaliaram a capacidade da TC sem contraste em graduar a pancreatite $\operatorname{aguda}^{(\mathbf{4 8 , 4 9 )}}$. Nenhum dos dois utilizou tomógrafo helicoidal ou com multidetectores. Maier ${ }^{(49)}$ demonstrou, em 90 pacientes, correlação estatisticamente significante entre o infiltrado inflamatório extrapancreático e o volume da glândula, avaliados pela TC sem contraste, com a gravidade da pancreatite aguda. Elias Júnior ${ }^{(48)}$, em sua tese de doutorado, comparou retrospectivamente os achados da TC sem contraste com os achados da TC com contraste, bem como com a evolução de 95 pacientes com pancreatite aguda. Demonstrou correlação positiva e estatisticamente significante entre o índice morfológico de Balthazar ${ }^{(63)}$, calculado pela TC sem contraste, com a taxa de mortalidade, tempo de jejum, bem como com o ISTC $^{(64)}$ e o grau de necrose demonstrados pela TC com contraste.

Efeitos deletérios diretos do contraste iodado IV sobre o pâncreas em pacientes
Figura 14. Paciente com pancreatite aguda, notando-se volumosa coleção em loja pancreática com imagens gasosas de permeio, indicando infecção (grau E do índice morfológico de Balthazar).

com pancreatite aguda têm sido apontados por alguns autores em estudos experimentais $^{(70,71)}$ e em humanos ${ }^{(72)}$. Somando-se aos outros efeitos deletérios desta substância já bem conhecidos (nefrotoxicidade, potencial alérgico, e outros), além da preocupação com a redução de custos, tempo do exame, com a dose de radiação em exames seriados muitas vezes necessários, o questionamento do papel da necrose pancreática no estadiamento da pancreatite aguda e o retardo de 48-72 horas para sua avaliação acurada, tem-se proposto o uso da TC sem contraste IV na avaliação de alterações peripancreáticas e da morfologia pancreática como alternativa para estratificação da gravidade da pancreatite agu-

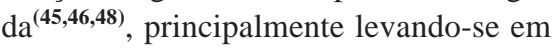
conta que os tomógrafos modernos helicoidais e com multidetectores, ainda não avaliados para este fim na literatura, têm melhor qualidade de imagem que os aparelhos convencionais $^{(48)}$.

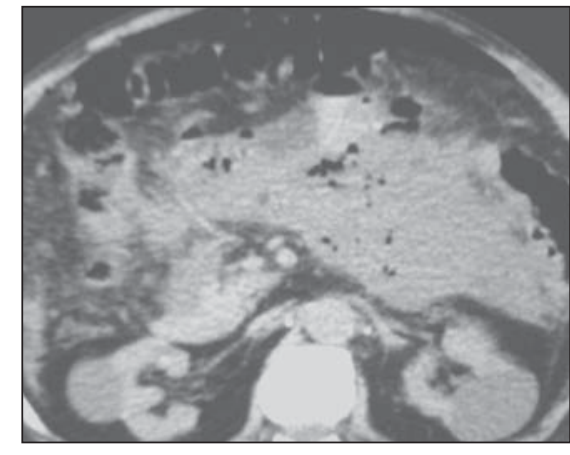




\section{APENDICITE EPIPLÓICA PRIMÁRIA}

A apendicite epiplóica é um processo inflamatório intra-abdominal infreqüente, atribuído a torção ou trombose venosa espontânea de um apêndice epiplóico, com conseqüente infarto isquêmico e inflamação ${ }^{(50)}$. A apresentação clínica é de dor abdominal focal aguda, sem quaisquer outras queixas ou sinais clínicos, sendo difícil a sua diferenciação de apendicite, diverticulite ou de outros processos abdômino-pélvicos agudos.

Na maioria dos casos a TC apresenta achados patognomônicos, caracterizados por lesão ovalada pericólica (com diâmetro entre 1 e $4 \mathrm{~cm}$ ), com densidade de gordura (mais densa que a gordura não envolvida), espessamento do peritônio visceral e estriações densas na gordura adjacente ao apêndice inflamado ${ }^{(51)}$. Achados tomográficos adicionais incluem um ponto central hiperatenuante e espessamento do peritônio parietal adjacente, bem como efeito de massa, espessamento de parede do cólon adjacente ou ambos $^{(\mathbf{5 0})}$ (Figuras 2, 10 e 11).

Esses achados são prontamente reconhecidos na TC espiral sem contraste IV, tendo sido esta técnica utilizada no estudo evolutivo realizado por Rao et al. ${ }^{(\mathbf{5 0})}$.

A identificação dessa anormalidade, que é autolimitada e benigna, evita intervenções cirúrgicas desnecessárias, o que justifica os esforços para a sua caracterização por meio da TC.

\section{PNEUMOPERITÔNIO}

A demonstração de pneumoperitônio em um paciente apresentando dor abdominal aguda constitui tarefa de grande impor- tância. Em mais de $90 \%$ dos casos a causa do pneumoperitônio necessitará de abordagem cirúrgica de emergência ${ }^{(73)}$.

A radiografia simples é muito sensível na detecção de ar livre intracavitário, contudo, a TC é claramente superior, especialmente em pacientes muito debilitados ou obesos, incapazes de cooperar com o exame radiográfico simples ${ }^{(\boldsymbol{6})}$. Entretanto, é defendida a idéia de que a superioridade da TC sobre a radiografia simples não é suficiente para justificar o abandono desta última, e que a menor detecção de pneumoperitônio pela radiografia é mais em função de negligência técnica do que de limitação física intrínseca do método ${ }^{(\mathbf{1})}$.

A despeito dessas considerações, em pacientes com coleções gasosas loculadas ou que foram submetidos a cirurgia, a radiografia é menos sensível, e ainda a TC é superior na detecção de ar extraluminal na parede intestinal ou no interior dos sistemas venosos mesentérico ou portal ${ }^{(6)}$. Para isto, não é necessária a utilização de contraste IV, como é facilmente compreensível (Figuras 8, 9 e 15).

É importante observar que a TC permite identificar o local e a causa da perfuração em apenas um terço dos casos, não devendo ser este o foco das nossas atenções ${ }^{(74)}$.

\section{OBSTRUÇÃO INTESTINAL}

A obstrução intestinal é responsável por aproximadamente $20 \%$ dos casos de abdome agudo ${ }^{(2)}$.

Existem controvérsias consideráveis sobre a melhor abordagem para a avaliação desses casos. Foi demonstrado que a radiografia simples ${ }^{(75)}$ ou a contrastada ${ }^{(76)}$ e a TC apresentam sensibilidade, especificidade e acurácia similares na identificação dos vários graus de obstrução. Entretanto, a TC mostrou ser o método mais rápido ${ }^{(76)}$ e mais eficaz na identificação da causa da obstrução, apresentando menor sensibilidade nos casos de baixo grau ou obstrução intermitente, nos quais a enteróclise apresenta bons resultados ${ }^{(77)}$. Ahn et al. ${ }^{(60)}$ demonstraram maior sensibilidade da TC em relação à radiografia simples na identificação da obstrução intestinal $(75 \% \times$ 49\%), porém, com especificidade e acurácia semelhantes $(99 \% \times 98 \%$ e $98 \% \times$ $96 \%$, respectivamente). No entanto, é importante observar que esses resultados podem ter sido decorrentes de um número muito reduzido de pacientes com obstrução intestinal comprovada $(n=3)$.

O exame tomográfico sem contrate $\mathrm{VO}$ pode ser realizado em grande parte dos pacientes com obstrução intestinal, nos quais há acúmulo de líquido intraluminal. A presença desse líquido funciona como um contraste natural, e assim o exame pode ser realizado imediatamente, evitando o atraso do preparo VO. Os sinais tomográficos de obstrução intestinal são a dilatação das alças de delgado, com calibre superior a $2,5 \mathrm{~cm}$, nível hidro-aéreo no seu interior e desproporção com segmento de alça distal com calibre normal indicando o nível da obstrução ${ }^{(77)}$ (Figura 16). A utilização destes sinais prescinde de contraste IV e VO. O uso de meio de contraste IV não foi discutido na literatura, e alguns autores que advogam o uso da TC utilizaram-no em seus trabalhos em virtualmente todos os pacientes ${ }^{(\mathbf{4 4}, 77)}$.

Diante dessas controvérsias, e considerando-se o fato de o exame radiográfico simples permitir o diagnóstico em grande número dos $\operatorname{casos}^{(\mathbf{7 5})}$, parece prematura a preconização da TC sem contraste IV como

Figura 15. Paciente com quadro de dor abdominal aguda, sendo caracterizada, em $\mathbf{A}$, pequena quantidade de ar em espaço subfrênico direito (setas), melhor demonstrada em $\mathbf{B}$, com janela para pulmão, refletindo abdome agudo perfurativo.
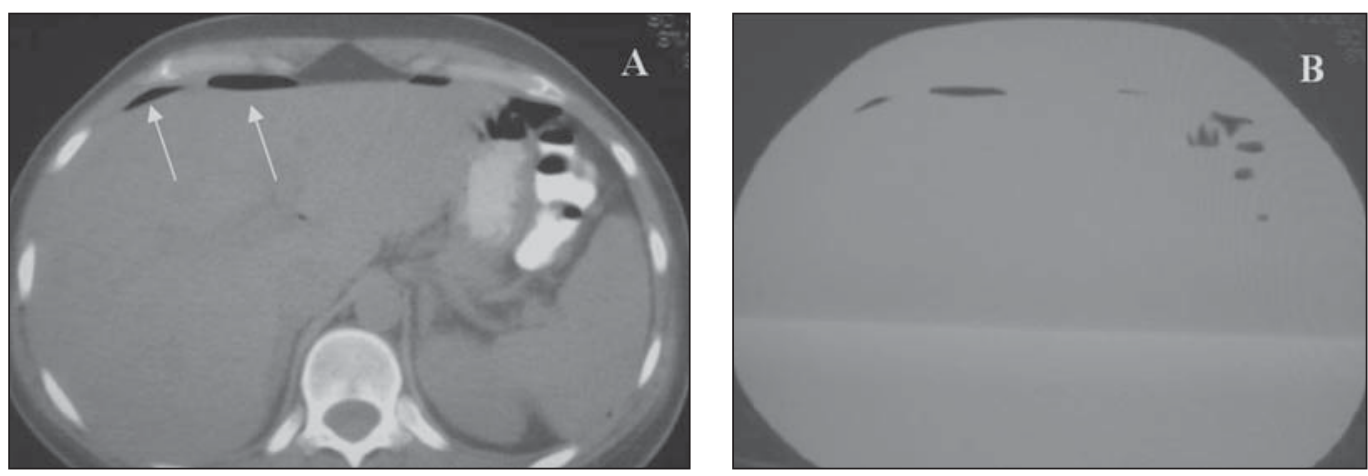


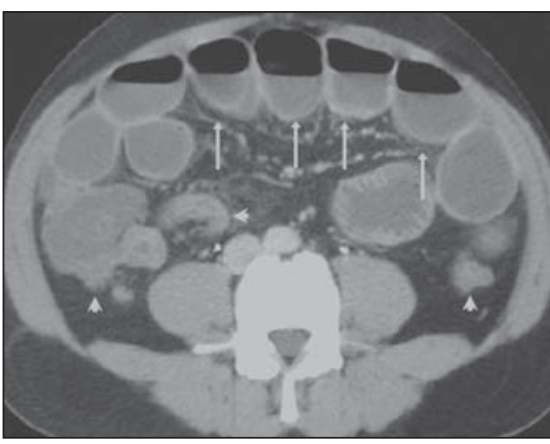

Figura 16. Obstrução intestinal. Dilatação de alças de delgado com níveis hidro-aéreos (setas), notando-se alças de íleo distal e cólon ascendente e descendente com calibre normal (cabeças de setas), demonstrando obstrução ao nível de íleo proximal por aderência (não demonstrada nesta figura).

método de avaliação inicial na obstrução intestinal. Essa possibilidade não pode, contudo, ser descartada, e o seu uso racional merece espaço na propedêutica radiológica atual, principalmente substituindo progressivamente o uso do trânsito intestinal, neste grupo de pacientes.

\section{INFARTO OMENTAL}

Um dos diagnósticos diferenciais de apendicite e diverticulite aguda é o infarto omental segmentar, pois, conforme a sua localização, apresenta sintomas semelhantes aos daquelas entidades ${ }^{(\mathbf{7 8 , 7 9 )}}$. O infarto omental segmentar ocorre por suprimento vascular inadequado de uma pequena porção do omento, geralmente localizada entre o cólon descendente ou ascendente e o peritônio ${ }^{(79)}$.

$\mathrm{Na}$ TC é possível identificar uma área fusiforme ou ovóide hiperdensa em relação à gordura mesenterial normal e apresentando algumas estrias densas de permeio e alterações inflamatórias (Figura 17). O cólon subjacente pode apresentar sinais de compressão extrínseca ou mostrar aspecto normal $^{(79,80)}$

A identificação dos sinais tomográficos supramencionados não é dependente da injeção IV de contraste, o que sugere que o diagnóstico de infarto omental seja possível através da TC sem contraste.

\section{CONCLUSÃO}

O termo abdome agudo possui um sentido amplo, englobando entidades distin-
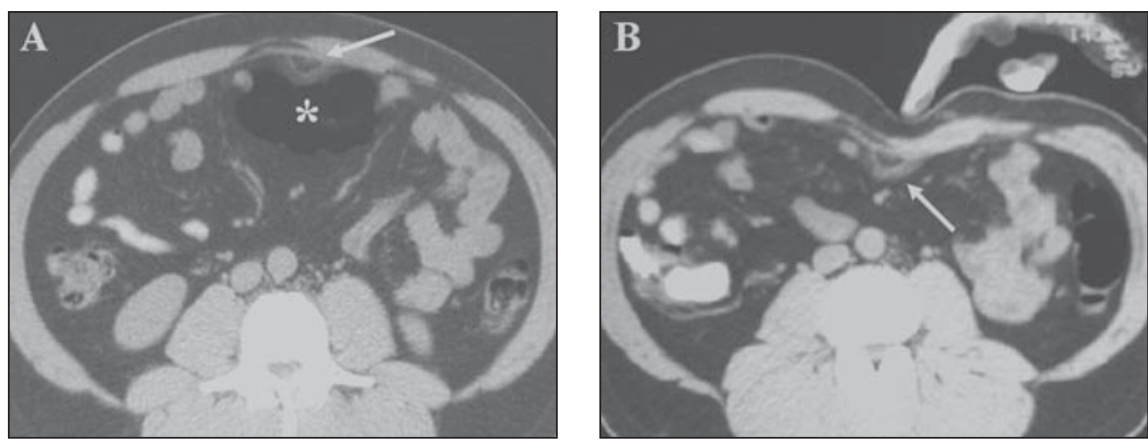

Figura 17. Infarto omental. Em A nota-se pequena imagem ovalada (seta) com densidade de gordura, porém mais densa que a gordura adjacente, localizada junto ao cólon transverso (asterisco), podendo corresponder a apendicite epiplóica ou pequeno infarto omental. Em B, após compressão digital do abdome com deslocamento do cólon transverso, a imagem ovalada (seta) permanece no mesmo local, afastando a hipótese de apendicite epiplóica.

tas, sejam funcionais ou morfológicas, que requerem atenção clínica ou cirúrgica imediata. A dor abdominal aguda está entre os três sintomas mais comuns em pacientes admitidos em serviços de emergência e hospitais $^{(78)}$.

Mindelzun et al. ${ }^{(6)}$ indicaram, a partir de dados de revisão obtidos pelo Comitê de Pesquisa da Organização Mundial de Gastroenterologia, que cerca de dois terços dos pacientes com dor abdominal aguda possuem uma anormalidade que pode ser identificada por intermédio de estudos de imagem.

A abordagem tradicional através de estudos radiológicos simples, contrastados ou da US ainda é predominante. Entretanto, a introdução da TC espiral sem contraste tem aos poucos modificado essa conduta, pois combina a rapidez do exame radiológico simples com alta resolução espacial e contraste entre partes moles.

A sua utilização apresenta grande impacto na determinação da causa e na orientação terapêutica do paciente com dor abdominal aguda, principalmente naqueles com forte suspeita clínica ${ }^{(78)}$

A qualidade das imagens obtidas através da TC sem contraste é inquestionavelmente melhor quando comparada à radiografia simples, e a literatura tem indicado, com poucas ressalvas, ganhos diagnósticos significativos que justificam a sua utilização, com destaque na suspeita de apendicite aguda, cólica nefrética e diverticulite.

Permanecem, contudo, em discussão, as questões sobre a disponibilidade de equipamentos e pessoal capacitado, bem como sobre os custos operacionais envolvidos, para que se proceda a uma adequação progressiva, racional e fundamentada dos algoritmos diagnósticos vigentes.

\section{REFERÊNCIAS}

1. Baker SR. Unenhanced helical CT versus plain abdominal radiography: a dissenting opinion. Radiology 1997;205:45-47.

2. Balthazar EJ, Birnbaum BA, Yee J, Megibow AJ, Roshkow J, Gray C. Acute appendicitis: CT and US correlation in 100 patients. Radiology 1994;190: 31-35

3. D'Ippolito G, de Melo GGN, Szejnfeld J. The value of unenhanced CT in the diagnosis of acute appendicitis. São Paulo Med J 1998;116:1838-1845.

4. Lane MJ, Katz DS, Ross BA, Clautice-Engle TL, Mindelzun RE, Jeffrey RB Jr. Unenhanced helical CT for suspected acute appendicitis. AJR 1997; 168:405-409.

5. Malone AJ Jr, Wolf CR, Malmed AS, Melliere BF Diagnosis of acute appendicitis: value of unenhanced CT. AJR 1993;160:763-766.

6. Mindelzun RE, Jeffrey RB. Unenhanced helical CT for evaluating acute abdominal pain: a little more cost, a lot more information. Radiology 1997;205: 43-45

7. Rao PM, Rhea JT, Novelline RA, Mostafavi AA Lawrason JN, McCabe CJ. Helical CT combined with contrast material administered only through the colon for imaging of suspected appendicitis. AJR 1997;169:1275-1280.

8. Peck J, Peck A, Peck C. The clinical role of noncontrast helical computed tomography in the diagnosis of acute appendicitis. Am J Surg 2000;180: 133-136.

9. Funaki B, Grosskreutz SR, Funaki CN. Using unenhanced helical CT with enteric contrast material for suspected appendicitis in patients treated at a community hospital. AJR 1998;171:997-1001.

10. Lane MJ, Liu DM, Huynh MD, Jeffrey RB Jr, Mindelzun RE, Katz DS. Suspected acute appendicitis: nonenhanced helical CT in 300 consecutive patients. Radiology 1999;213:341-346.

11. Mullins ME, Kircher MF, Ryan DP, et al. Evaluation of suspected appendicitis in children using lim- 
ited helical CT and colonic contrast material. AJR 2001;176:37-41

12. Ege G, Akman H, Sahin A, Bugra D, Kuzucu K. Diagnostic value of unenhanced helical CT in adult patients with suspected acute appendicitis. Br J Radiol 2002;75:721-725.

13. Weyant MJ, Barie PS, Eachempati SR. Clinical role of noncontrast helical computed tomography in diagnosis of acute appendicitis. Am J Surg 2002; 183:97-99.

14. Rao PM, Rhea JT, Novelline RA. Helical CT of appendicitis and diverticulitis. Radiol Clin North Am 1999;37:895-910.

15. Novelline RA, Rhea JT, Rao PM, Stuk JL. Helical CT in emergency radiology. Radiology 1999;213: 321-339.

16. Fielding JR, Fox LA, Heller H, et al. Spiral CT in the evaluation of flank pain: overall accuracy and feature analysis. J Comput Assist Tomogr 1997;21: 635-638.

17. Smith RC, Rosenfield AT, Choe KA, et al. Acute flank pain: comparison of non-contrast-enhanced CT and intravenous urography. Radiology 1995; 194:789-794.

18. Smith RC, Verga M, McCarthy S, Rosenfield AT. Diagnosis of acute flank pain: value of unenhanced helical CT. AJR 1996;166:97-101.

19. Sommer FG, Jeffrey RB Jr, Rubin GD, et al. Detection of ureteral calculi in patients with suspected renal colic: value of reformatted noncontrast helical CT. AJR 1995;165:509-513.

20. Varanelli MJ, Coll DM, Levine JA, Rosenfield AT Smith RC. Relationship between duration of pain and secondary signs of obstruction of the urinary tract on unenhanced helical CT. AJR 2001;177: 325-330.

21. Colistro R, Torreggiani WC, Lyburn ID, et al. Unenhanced helical CT in the investigation of acute flank pain. Clin Radiol 2002;57:435-441.

22. Catalano O, Nunziata A, Altei F, Siani A. Suspected ureteral colic: primary helical CT versus selective helical CT after unenhanced radiography and sonography. AJR 2002;178:379-387.

23. Hamm M, Knopfle E, Wartenberg S, Wawroschek F, Weckermann D, Harzmann R. Low dose unenhanced helical computerized tomography for the evaluation of acute flank pain. J Urol 2002;167: 1687-1691.

24. Gottlieb RH, La TC, Erturk EN, et al. CT in detecting urinary tract calculi: influence on patient imaging and clinical outcomes. Radiology 2002;225: 441-449.

25. Freed KS, Paulson EK, Frederick MG, et al. Interobserver variability in the interpretation of unenhanced helical CT for the diagnosis of ureteral stone disease. J Comput Assist Tomogr 1998;22: 732-737.

26. Sourtzis S, Thibeau JF, Damry N, Raslan A, Vandendris M, Bellemans M. Radiologic investigation of renal colic: unenhanced helical CT compared with excretory urography. AJR 1999;172:14911494.

27. Dalrymple NC, Casford B, Raiken DP, Elsass KD, Pagan RA. Pearls and pitfalls in the diagnosis of ureterolithiasis with unenhanced helical CT. RadioGraphics 2000;20:439-447; quiz 527-528, 532.

28. Georgiades CS, Moore CJ, Smith DP. Differences of renal parenchymal attenuation for acutely obstructed and unobstructed kidneys on unenhanced helical CT: a useful secondary sign? AJR 2001; 176:965-968.
29. Katz DS, Scheer M, Lumerman JH, Mellinger BC, Stillman CA, Lane MJ. Alternative or additional diagnoses on unenhanced helical computed tomography for suspected renal colic: experience with 1000 consecutive examinations. Urology 2000;56: $53-57$.

30. Denton ER, Mackenzie A, Greenwell T, Popert R, Rankin SC. Unenhanced helical CT for renal colic - is the radiation dose justifiable? Clin Radiol 1999;54:444-447.

31. Rao PM, Rhea JT, Novelline RA, et al. Helical CT with only colonic contrast material for diagnosing diverticulitis: prospective evaluation of 150 patients. AJR 1998;170:1445-1449.

32. Jeffrey RB. Imaging the acute abdome: the impact of computed tomography and sonography. In: Gore MR, Levine MS, editors. Textbook of gastrointestinal radiology. 2nd ed. Philadelphia, Pa: WB Saunders, 2000;2186-2195.

33. Kircher MF, Rhea JT, Kihiczak D, Novelline RA. Frequency, sensitivity, and specificity of individual signs of diverticulitis on thin-section helical CT with colonic contrast material: experience with 312 cases. AJR 2002;178:1313-1318.

34. Galvão Filho MM, D'Ippolito G, Hartmann LG, et al. O valor da tomografia computadorizada helicoidal sem contraste na avaliação de pacientes com dor no flanco. Radiol Bras 2001;34:129-134.

35. D'Ippolito G, Nunes Jr JAT, Wolosker AMB, Borri ML, Anderaus S, Szejnfeld J. O valor da tomografia computadorizada sem contraste na avaliação da região cecoapendicular normal. Radiol Bras 1996; 29:247-251.

36. Lanoue MZ, Mindell HJ. The use of unenhanced helical CT to evaluate suspected renal colic. AJR 1997;169:1579-1584.

37. Napel AS. Basic principles of spiral CT. In: Fishman EK, Jeffrey RB Jr, editors. Spiral CT: principles, techniques, and clinical applications. Philadelphia, PA: Lippincott-Raven, 1996.

38. Raptopoulos V, Katsou G, Rosen MP, Siewert B, Goldberg SN, Kruskal JB. Acute appendicitis: effect of increased use of CT on selecting patients earlier. Radiology 2003;226:521-526.

39. Rhea JT, Rao PM, Novelline RA, McCabe CJ. A focused appendiceal CT technique to reduce the cost of caring for patients with clinically suspected appendicitis. AJR 1997;169:113-118.

40. Cho KC, Morehouse HT, Alterman DD, Thornhill BA. Sigmoid diverticulitis: diagnostic role of CT - comparison with barium enema studies. Radiology 1990;176:111-115.

41. Padidar AM, Jeffrey RB Jr, Mindelzun RE, Dolph JF. Differentiating sigmoid diverticulitis from carcinoma on CT scans: mesenteric inflammation suggests diverticulitis. AJR 1994;163:81-83.

42. Herlinger H, Rubesin SE. Obstruction. In: Gore RM, Levine MS, Laufer I, editors. Textbook of gastrointestinal radiology. 1st ed. Philadelphia, Pa: WB Saunders, 1994.

43. Lewis FR, Holcroft JW, Boey J, Dunphy JE. Appendicitis: a critical review of diagnosis and treatment in 1000 cases. Arch Surg 1975;110:677-684.

44. Taourel PG, Fabre JM, Pradel JA, Seneterre EJ, Megibow AJ, Bruel JM. Value of CT in the diagnosis and management of patients with suspected acute small-bowel obstruction. AJR 1995;165: 1187-1192.

45. Schröder T, Kivisaari L, Somer K, StandertskjoldNordenstam CG, Kivilaakso E, Lempinen M. Significance of extrapancreatic findings in computed tomography (CT) of acute pancreatitis. Eur J Radiol 1985;5:273-275

46. De Sanctis JT, Lee MJ, Gazelle GS, et al. Prognostic indicators in acute pancreatitis: CT vs APACHE II. Clin Radiol 1997;52:842-848.

47. Balthazar EJ. Acute pancreatitis: assessment of severity with clinical and CT evaluation. Radiology 2002;223:603-613.

48. Elias Júnior J. Utilização da tomografia computadorizada sem contraste e da ressonância magnética no diagnóstico e na estratificação da gravidade da pancreatite aguda. (Tese de Doutorado). Ribeirão Preto, SP: Universidade de São Paulo - Faculdade de Medicina de Ribeirão Preto, 2002.

49. Maier W. The value of non-contrast computed tomography in acute pancreatitis. Rofo 1989;150: $458-461$.

50. Rao PM, Wittenberg J, Lawrason JN. Primary epiploic appendagitis: evolutionary changes in CT appearance. Radiology 1997;204:713-717.

51. Rioux M, Langis P. Primary epiploic appendagitis: clinical, US, and CT findings in 14 cases. Radiology 1994;191:523-526.

52. Siewert B, Raptopoulos V. CT of the acute abdomen: findings and impact on diagnosis and treatment. AJR 1994;163:1317-1324.

53. Berry J Jr, Malt RA. Appendicitis near its centenary. Ann Surg 1984;200:567-575.

54. Puylaert JB, Rutgers PH, Lalisang RI, et al. A prospective study of ultrasonography in the diagnosis of appendicitis. N Engl J Med 1987;317:666-669.

55. Balthazar EJ, Megibow AJ, Siegel SE, Birnbaum BA. Appendicitis: prospective evaluation with high-resolution CT. Radiology 1991;180:21-24.

56. Jacobs JE, Birnbaum BA, Macari M, et al. Acute appendicitis: comparison of helical CT diagnosis focused technique with oral contrast material versus nonfocused technique with oral and intravenous contrast material. Radiology 2001;220:683-690.

57. Jacobs JE, Birnbaum BA. Computed tomography evaluation of acute pancreatitis. Semin Roentgenol 2001;36:92-98.

58. Hubner WA, Irby P, Stoller ML. Natural history and current concepts for the treatment of small ureteral calculi. Eur Urol 1993;24:172-176.

59. Balthazar EJ. Diverticular disease. In: Gore RM, Levine MS, Laufer I, editors. Textbook of gastrointestinal radiology. Philadelphia, Pa: WB Saunders, 1994

60. Ahn SH, Mayo-Smith WW, Murphy BL, Reinert SE, Cronan JJ. Acute nontraumatic abdominal pain in adult patients: abdominal radiography compared with CT evaluation. Radiology 2002;225:159164.

61. Jang HJ, Lim HK, Lee SJ, Lee WJ, Kim EY, Kim $\mathrm{SH}$. Acute diverticulitis of the cecum and ascending colon: the value of thin-section helical CT findings in excluding colonic carcinoma. AJR 2000; 174:1397-1402.

62. Rall PW, Bree RL, Glick SN, et al. Acute pancreatitis. Reston, Va: American College of Radiology, 2001.

63. Balthazar EJ, Ranson JH, Naidich DP, Megibow AJ, Caccavale R, Cooper MM. Acute pancreatitis: prognostic value of CT. Radiology 1985;156:767772 .

64. Balthazar EJ, Robinson DL, Megibow AJ, Ranson $\mathrm{JH}$. Acute pancreatitis: value of CT in establishing prognosis. Radiology 1990;174:331-336.

65. Simchuk EJ, Traverso LW, Nukui Y, Kozarek RA. 
Computed tomography severity index is a predictor of outcomes for severe pancreatitis. Am J Surg 2000;179:352-355.

66. Dervenis C, Johnson CD, Bassi C, et al. Diagnosis, objective assessment of severity, and management of acute pancreatitis. Santorini consensus conference. Int J Pancreatol 1999;25:195-210.

67. Balthazar EJ, Freeny PC. Contrast-enhanced computed tomography in acute pancreatitis: is it beneficial or harmful? Gastroenterology 1994;106: 259-262.

68. van den Biezenbos AR, Kruyt PM, Bosscha K, et al. Added value of CT criteria compared to the clinical SAP score in patients with acute pancreatitis. Abdom Imaging 1998;23:622-626.

69. London NJ, Leese T, Lavelle JM, et al. Rapid-bolus contrast-enhanced dynamic computed tomography in acute pancreatitis: a prospective study. $\mathrm{Br}$ J Surg 1991;78:1452-1456.

70. Schmidt J, Hotz HG, Foitzik T, et al. Intravenous contrast medium aggravates the impairment of pancreatic microcirculation in necrotizing pancreatitis in the rat. Ann Surg 1995;221:257-264.

71. Foitzik T, Bassi DG, Schmidt J, et al. Intravenous contrast medium accentuates the severity of acute necrotizing pancreatitis in the rat. Gastroenterology 1994;106:207-214.

72. McMenamin DA, Gates LK Jr. A retrospective analysis of the effect of contrast-enhanced CT on the outcome of acute pancreatitis. Am J Gastroenterol 1996;91:1384-1387.

73. Field S. The acute abdomen. In: Sutton D, editor. Textbook of radiology and medical imaging. New York, NY: Churchill Livingstone, 1992.

74. Chen CH, Huang HS, Yang CC, Yeh YH. The features of perforated peptic ulcers in conventional computed tomography. Hepatogastroenterology 2001;48:1393-1396.

75. Maglinte DD, Reyes BL, Harmon BH, et al. Reliability and role of plain film radiography and $\mathrm{CT}$ in the diagnosis of small-bowel obstruction. AJR 1996; $167: 1451-1455$.

76. Bogusevicius A, Maleckas A, Pundzius J, Skaudickas D. Prospective randomised trial of computer-aided diagnosis and contrast radiography in acute small bowel obstruction. Eur J Surg 2002; 168:78-83.

77. Balthazar EJ. George W. Holmes Lecture. CT of small-bowel obstruction. AJR 1994;162:255-261.

78. Siewert B, Raptopoulos V, Mueller MF, Rosen MP, Steer M. Impact of CT on diagnosis and management of acute abdomen in patients initially treated without surgery. AJR 1997;168:173-178.

79. Crofoot DD. Spontaneous segmental infarction of the greater omentum. Am J Surg 1980;139:262264.

80. Moreira LBM, Pinheiro RA, Melo ASA, Alves JRD, Noro F, Marchiori E. Paniculite mesentérica: aspectos na tomografia computadorizada. Radiol Bras 2001;34:135-140. 